\title{
Mathematical Solution and Simulation for Detection Location of Bus Signal Priority
}

\author{
Jaeseok Kwon ${ }^{1}$, Youngje Jeong ${ }^{2, *}$, Hyerim $\mathrm{Cho}^{3}$ and Youngchan $\mathrm{Kim}^{1}$ \\ ${ }^{1}$ University of Seoul, 90 Jeonnong-dong, Dongdaemun-gu, Seoul, Korea \\ ${ }^{2}$ Korea Road Traffic Authority, 2 Hyeoksin-ro, Wonju, Korea \\ ${ }^{3}$ Seoul Metropolitan Government, 15 Deoksugung-gil, Jung-gu, Seoul, Korea \\ ${ }^{*}$ Corresponding author
}

\begin{abstract}
This study suggests new mathematical solution and simulation results for detection location of bus signal priority. This mathematical model can determine the detector location for the minimum bus delay. With the application of the bus speed and detector location, this study analyzed the change of bus delay. In addition, this study analyzed the bus signal priority using a microscopic simulation under the same conditions with the analysis of bus waiting time. The result of microscopic simulation using VISSIM also showed the similar trend with the results from the waiting time model. This research made sure that the bus delay can be changed according to the detector location only, even if the delay of passenger car had a slight change.
\end{abstract}

Keywords-ppriority; detector; mathematic solution; simulation

\section{INTRODUCTION}

The bus signal priority is a signal control strategy for improving the mobility of bus at a signalized intersection. It is classified into two types--priority and preemption--depending on the force of the phase operation. In general, the bus signal priority has a bad effect on the delay of passenger car. So, the recent studies focus on a combination of the real-time traffic signal control and the bus signal priority for improving the passenger car delay which has increased as a result of the bus signal priority. Recently, the transit priority facilities such as the median bus lane have been expanded to improve the mobility of bus in Seoul. Alongside this, various kinds of researches related to the improvement of the transit operation have been presented to improve the level of bus service.

The bus signal priority is separated into the active and passive priority strategy. The active bus signal priority can provide the right-of-way opportunity for a bus based on a real time detection system. The early green which performs the early start of bus phase and the green extension which carries out the extension of bus signal are the most typical active bus priority strategies. Various factors including the detector location, the time length of priority and non-priority phase and the operation method of priority phase influence on the operation strategy for the active bus signal priority. The detector in active bus signal priority is located about $80-150 \mathrm{~m}$ behind the stop line according to an empirical method. [1] This study suggested the determination method of detector location that can minimize the bus delay by modeling the process of active bus signal priority on a median bus lane. This study carried out the calculation model of the bus waiting time based on the active bus signal priority. The most common active priority techniques such as the early green and green extension were singled out as a priority method of this study.

This research selected the exclusive median bus lane as the application object of the active bus signal priority. So, we suggested a phase operation method for the thru phase in the median bus lane and the left-turn phase in the general-purpose lane which cannot overlap. We showed the calculation model of bus waiting time from the macroscopic view and a process of active bus signal priority according to the bus detection time. This study analyzed the effects of the bus waiting time model through the case study that has the various traffic signal timings and the free flow speed of bus.

\section{LITERATURE REVIEW}

In active bus signal priority, the detector location is generally around $80-150 \mathrm{~m}$ behind the stop line. The active bus signal priority of L.A., U.S. is about 10 seconds for the early green and green extension. The detector location is considered the bus speed for the early green and green extension. [2] In Sacramento, U.S. about 10 seconds for early green and green extension time is applied with a detector location of 300-600ft. [3] In Toronto, Canada, the time length for early green and green extension corresponds to about 16 seconds for major intersections, and an ordinary intersection need about 30 seconds for priority phases. The detector is installed within $100 \mathrm{~m}$ for a priority request. In Melbourne, Australia, an early green and green extension time corresponds to $20 \%$ of the cycle time, and the detector is installed about $200 \mathrm{~m}$ away behind the stop line to collect bus location information. [4] The similar study with this research suggests the relation the optimal detector location and the bus waiting time based on the calculation model. This study uses the priority strategy that provides the all variable green time of non-priority phases with the priority phase. The priority phase can use the whole remaining time of phase excluding the minimum green time. Therefore, the green extension and the early green with the fixed priority time is not applied the bus waiting time model. [5] In addition, the study for the queue jump control which is similar to bus signal priority suggests the selection method of the detector location that can minimize the bus travel time at intersection where there is a queue jump exclusive lane. This study presents a maximum reduction of bus delay at $100-150 \mathrm{~m}$ based on the microscopic simulation. The simulation results for 
the optimal detector location of queue jump are similar to the results of the active bus signal priority. [6] A review of preceding studies about the detector location for the active bus signal priority pointed out the need to analyze about the relation of the signal priority process and the optimal detector location. This research shows the waiting time model of bus based on an early green and green extension with a fixed time length which is the most common active bus signal priority strategy. This study aims to develop the selection method of detector location for the active bus signal priority in the median bus lane. The new method can determine the detector location to minimize the bus delay of median bus lane without influencing passenger car delay of general-purpose lane.

\section{MATHEMATiCAl SOLUTION FOR DETECTION LOCATION}

This study suggested the calculation model of bus waiting time according to the bus detection time to select the optimal detector location for the active bus signal priority. This model can determine a detector location that can minimize the waiting time of bus and can calculate the bus waiting time at the signalized intersection based on the bus detection time, the current phase order and variable green time under the active bus signal priority condition. This model has the some assumptions that a bus drives at a free flow speed and on a median bus lane. In addition, a bus travels at under-saturated condition, and this model does not consider the acceleration and deceleration of bus.

The first case is the model for green extension. If the bus is detected during its thru phase, the range of bus waiting time is from 0 to the total red time as shown in equation (1). The range of bus waiting time depends on whether or not the bus passed the intersection as shown in equation (3). The maximum waiting time of bus consists of the recalculated variable green time for green extension, the minimum green time and the yellow. When the bus does not pass the intersection even if bus signal priority was provided, the bus can experience the maximum waiting time. The remaining phases are recalculated using the split ratio in the normal condition as shown in equation (4). This model can calculate the bus waiting time using a dummy variable $\alpha(\mathrm{t})$ as shown in equation (2)

The second case is the model for early green as shown in equation (5). When the bus is detected between the end of priority phase and the end of last non-priority phase, this model can be applied for the calculation of bus waiting time. The bus waiting time at the signalized intersection consists of the minimum green time and the yellow time of remaining phases from the bus detection time to the end of cycle, the recalculated variable green time and the available variable green time of current phase for the signal priority as shown in equation (6). The reduced time of current phase for early green can be determined by the comparison of the remaining time of current phase and the assigned time for early green. The reduced time of current phase can be determined directly by the dummy variable $\beta(t)$ as shown in equation (7). If the remaining time of current phase is shorter than the assigned time for early green, the current phase is forced off at once. In addition, this model considers the travel time of bus from the detector location to the stop line for the accurate bus waiting time. A dummy variable $\gamma(\mathrm{t})$ make the amber phase to keep the original time length as shown in equation (8), and this model can check the progress of amber phase using a dummy variable $\delta(t)$ as shown in equation (9). This model applies the active bus signal priority that uses the early green of the fixed time length, but each non-priority phase has the each reduced time depending on the bus detection time. This study suggests the calculation method of priority phase using the variable green time only excluding the minimum green and yellow times. Accordingly, the range of reduced time for the early green was from 0 to the total variable green time as shown in equation (11). The reduced non-priority phase for the early green is determined by the minimum green time of the remaining phases as shown in equation (10).

This waiting time model of bus can express the change of the priority and non-priority phase length under the active bus signal priority strategy depending on the bus detection time. This model separates the applied time period of the green extension and early green and applies the thru phase of bus for the starting point of a cycle as shown in Figure 1. This study suggests an equation for calculating the bus waiting time at intersections on all occasions. The bus waiting time means the time period from the arrival time to passing time at intersection after the bus detection. The expected value of the bus waiting time can be calculated by changing the detection time every second. It is expected that the efficiency of active bus signal priority can be maximized by selecting the detector location that has the minimized bus waiting time.

Case 1:

$$
0 \leq t \leq G_{j}
$$

$$
\begin{aligned}
& T(t)=\alpha(t)\left[G_{k}+A_{k}+E x t-T T_{B U S}-t\right. \\
& \left.+\sum_{i=k+1}^{n}\left(M i n G_{i}+\operatorname{Re} c G_{i}+A_{i}\right)\right]
\end{aligned}
$$

$$
\begin{aligned}
& \alpha(t)=\left\{\begin{array}{cc}
1 ; & \left(G_{k}+E X T-T T_{B U S}<t<\text { Cycle }\right) \\
0 ; & \text { others }
\end{array}\right. \\
& \operatorname{Rec} G_{i}=G_{i}-M i n G_{i}-\frac{G_{i}}{\sum_{i=j+1}^{n} G_{i}} E X T
\end{aligned}
$$

Case 2:

$$
G_{j}<t<\text { Cycle }
$$




$$
\begin{aligned}
T(t)=\sum_{i=k+1}^{n} & \left(\operatorname{MinG}_{i}+\operatorname{Re} c G_{i}+A_{i}\right) \\
& +\beta(t)\left(\sum_{i=1}^{k}\left(G_{i}+A_{i}\right)-E G \frac{G_{k}}{\sum_{i=k}^{n} G_{i}}-t\right) \\
& +\chi(t) A_{k} \\
& +\delta(t)\left(\sum_{i=1}^{k}\left(G_{i}+A_{i}\right)-t\right)-T T_{B U S}
\end{aligned}
$$

$$
\beta(t)=\left\{\begin{array}{cc} 
& \sum_{i=1}^{k-1}\left(G_{i}+A_{i}\right)<t \\
1 ; & <\sum_{i=1}^{k-1}\left(G_{i}+A_{i}\right)+G_{k}-E G \frac{G_{k}}{\sum_{i=k}^{n} G_{i}} \\
0 ; & \text { others }
\end{array}\right.
$$

$$
\chi(t)=\left\{\begin{array}{cc}
1 ; & \sum_{i=1}^{k-1}\left(G_{i}+A_{i}\right)+G_{k}-E G \frac{G_{k}}{\sum_{i=k}^{n} G_{i}}< \\
0 ; & \begin{array}{c}
t<\sum_{i=1}^{k-1}\left(G_{i}+A_{i}\right)+G_{k} \\
\text { others }
\end{array}
\end{array}\right.
$$

$$
\delta(t)=\left\{\begin{array}{rr}
1 ; & G_{j}<t \leq G_{j}+A_{j} \\
0 ; & \text { others }
\end{array}\right.
$$

$$
\operatorname{Re} c G_{i}=G_{i}-M i n G_{i}-\frac{G_{i}}{\sum_{i=j+1}^{n} G_{i}}\left(E G-R E G_{k}\right)
$$

Where;

$$
\begin{aligned}
& t: \text { Detection time of a bus (sec); } \\
& k \text { : Phase number at time t; } \\
& i \text { : Phase number; }
\end{aligned}
$$

$j$ : Phase number for the priority;

$T(t)$ : Waiting time of the bus that was detected at time t;

$G_{i}$ : Time length of phase i;

$\operatorname{Min}_{i}:$ Minimum green time of phase i;

$\operatorname{Re} c G_{i}:$ Re-calculated variable green time of phase $\mathrm{i}$;

$A_{i}:$ Amber of phase i;

Ext : Time length of green extension;

$E G$ : Time length of early green;

$\operatorname{Re} G_{k}$ : Decreased time length of phase k for early green;

$$
T T_{B U S}: \text { Travel time from detector to stop line }
$$

\section{SimUlation STUdY}

The bus waiting time model was assessed based on the case study. The analysis scenarios had the signal timing of two types and the isolated intersection of median bus lane as shown in table I. We designated the minimum and variable green time, split and priority phase for the analysis. The geometry is a fourleg intersection with a median bus lane as shown in Figure 1, and this research suggested the phase operation that the thru phase of median bus lane and the left-turn phase of generalpurpose lane can not be overlaped.

The traffic volume of general-purpose lane was applied to maintain service level D of the Korea Highway Capacity Manual to clearly identify the delay change. The bus traffic volume on the median bus lane was applied for 30 buses per hour, so the early green or the green extension can occur every second during cycle time. This research conducted the equation and microscopic simulation analysis by the free flow speed of bus and the detector location. In equation analysis, there was a rapid decline of the bus waiting from the stop line to the critical location of detector. In addition, the bus waiting time increased again beyond the critical location, but this re-increase trend of bus waiting time was insignificant in all scenarios. In scenario 1 , the minimum bus waiting time was observed at a detector location of 80-120 $\mathrm{m}$ as shown in Figure 2. In scenario 2 with an increased cycle length and traffic volume, the detector location for the minimized bus waiting time was an $80-140 \mathrm{~m}$ section as shown in Figure 3. The primary causes of these were an increase the frequency of the bus signal priority due to the shortening of cycle length and a change of the bus travel time from the detector to the stop line according to the free flow speed of bus. This analysis method is similar to a vertical queue of an input-output analysis and can calculate just the bus waiting time at intersection excluding the deceleration and acceleration of bus.

TABLE I. SIMULATION STUDY SCENARIO

\begin{tabular}{|c|c|c|c|c|c|}
\hline \multirow{2}{*}{ Scenario } & $\begin{array}{c}\text { Phase } \\
\text { Number }\end{array}$ & $\begin{array}{c}\text { Minimum } \\
\text { Green Time } \\
\text { (sec) }\end{array}$ & $\begin{array}{c}\text { Variable } \\
\text { Green Time } \\
\text { (sec) }\end{array}$ & $\begin{array}{c}\text { Green } \\
\text { Time } \\
\text { (sec) }\end{array}$ & $\begin{array}{c}\text { Yellow } \\
\text { (sec) }\end{array}$ \\
\hline \multirow{2}{*}{$\begin{array}{c}\text { Scenario } \\
\text { No.1 } \\
\text { Cycle } \\
\text { Length } \\
120 \text { sec }\end{array}$} & 1 (priority) & 10 & 22 & 32 & 3 \\
\cline { 2 - 6 } & 2(non-priority) & 10 & 12 & 22 & 3 \\
\cline { 2 - 6 } & 4(non-priority) & 10 & 22 & 32 & 3 \\
\hline \multirow{2}{*}{$\begin{array}{c}\text { Scenario } \\
\text { No.2 } \\
\text { Cycle }\end{array}$} & 1(priority) & 10 & 12 & 22 & 3 \\
\cline { 2 - 7 } $\begin{array}{c}\text { Length } \\
150 \text { sec }\end{array}$ & 3(non-priority) & 10 & 17 & 27 & 3 \\
\cline { 2 - 7 } & 4 (non-priority) & 10 & 17 & 27 & 3 \\
\hline
\end{tabular}




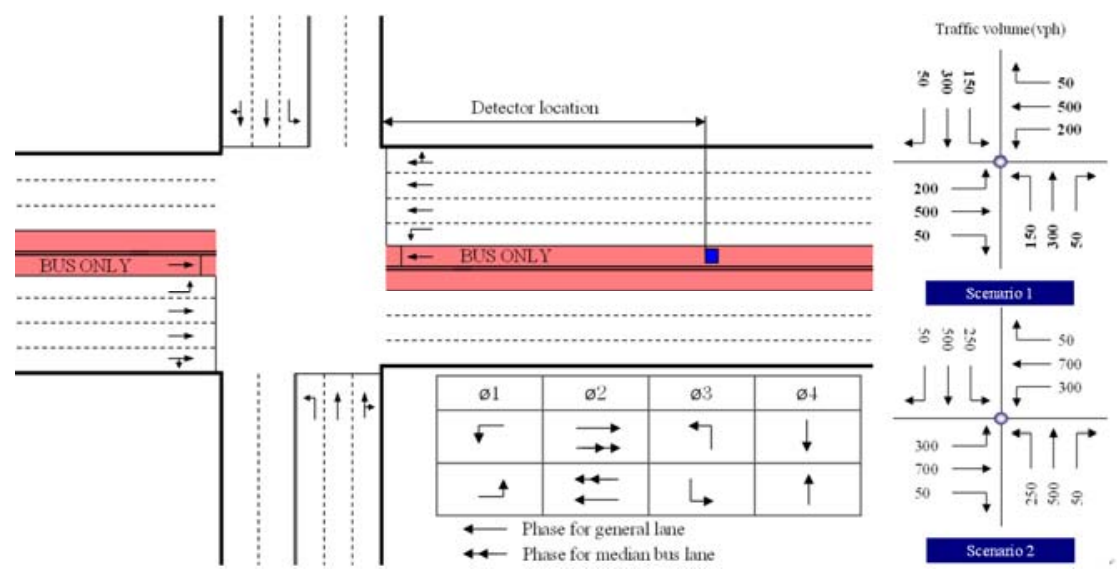

FIGURE I. GEOMETRY AND TRAFFIC CONDITION FOR SIMULATION STUDY

Together with the equation analysis of the bus waiting time, we conducted the microscopic simulation analysis using the VISSIM. While VISSIM provides its own bus signal priority function, this research used the VISVAP of VISSIM for the early green and green extension of the active bus signal priority on the median bus lane. As a result of the simulation analysis in the scenario 1, there was a rapid change in the bus delay of median bus lane until it reached the critical detector location which was similar to the analysis of bus waiting time. However, the passenger car delay of general-purpose lane had a low change of less than 2 seconds per vehicle due to the bus signal priority as shown in Figure 4. In addition, the simulation analysis showed a slight increase of the bus delay after a minimum delay occurred at a specific detector location as shown in Figure 5. In scenario 2, the bus delay increased as the detector location after the bus delay rapidly decreased up to the specific detector location such as in scenario 1 .

\section{CONCLUSION}

This research suggests the bus waiting time model to calculate the delay considering the detection time based on the active bus signal priority, and the users can determine the optimum detector location using this results. This research applies the most general green extension and early green for the priority strategy, and this model can calculate the time that bus has to wait at signal intersection in macroscopic view. The green extension and early green can have their own time period for the priority phase, so this research suggests the bus waiting time model for each priority function.

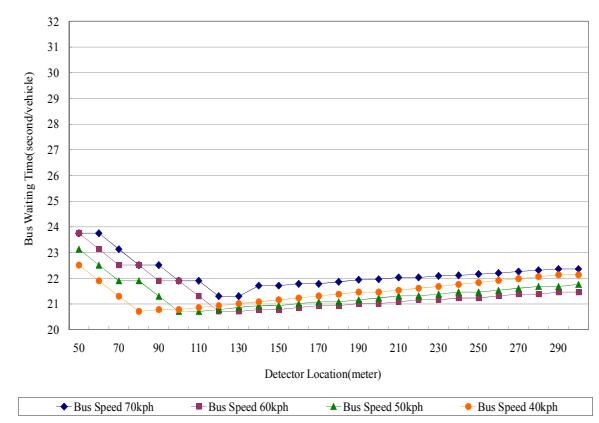

FIGURE II. BUS WAITING TIME OF SCENARIO 1

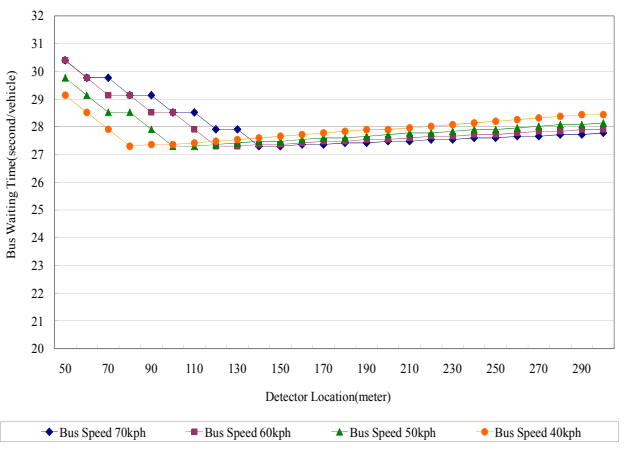

FIGURE III. BUS WAITING TIME OF SCENARIO 2

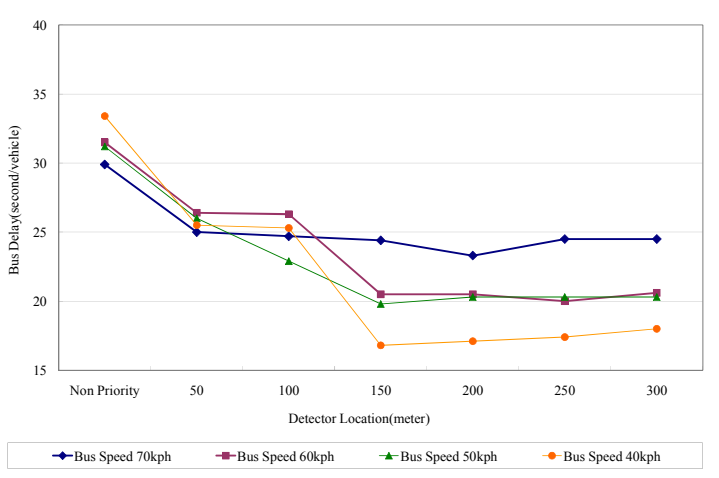

FIGURE IV. BUS DELAY OF SCENARIO 1

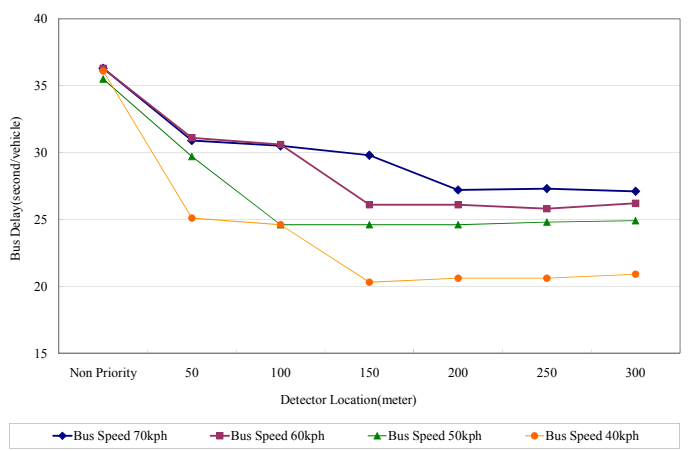

FIGURE V. BUS DELAY OF SCENARIO 2 


\section{REFERENCES}

[1] Harriet, R. Smith, and Brendon Hemily, "Transit Signal Priority: A Planning and Implementation Handbook", ITS America, 2005.

[2] Levinson, H.S., and Zimmerman, S., "TCRP 90, BUS RAPID TRANSIT Volume 1: Case Studies in Bus Rapid Transit", Transportation Research Board, National Research Council, Washington, D.C., 2003, pp.4-5.

[3] Rephlo, J., and R. Haas, "Sacramento-Watt Avenue Transit Priority and Mobility Enhancement Demonstration Project, Phase III Evaluation Report", U.S. Department of Transportation, Federal Highway Administration, Washington, D.C., 2006, pp.5-6.

[4] Currie, G., and A. Shalaby, "Active Transit Signal Priority for street cars - Experience in Melbourne, Australia and Toronto, Canada", Presented at the 87th Annual Meeting of the Transportation Research Board, Washington, D.C, 2008.

[5] Liu, H., A. Skabardonis, W. Zhang, and M. Li, "Optimal Detector Location for Bus Signal Priority", Transportation Research Record: Journal of the Transportation Research Board, Volume 1864, Transportation Research Board of the National Academies, Washington, D.C., 2004, pp.144-150.

[6] Zhou, G. W., and A. Gan, "Determination of Optimal Detector Location for Transit Signal Priority with Queue Jumper Lanes", Transportation Research Record: Journal of the Transportation Research Board, Volume 1978, Transportation Research Board of the National Academies, Washington, D.C., 2006, pp.123-129. 